\title{
Environment and Socio-Economic Influence of Victim of Malaria and Typhoid Fever in Nigeria
}

\author{
Yahaya Abdulkarim \\ Department of General Studies (GNS), Federal Polytechnics, Nasarawa, Nigeria.
}

\begin{abstract}
Malaria and typhoid fever are among the most endemic diseases in developing societies especially Nigeria. Both diseases have been associated with poverty and underdevelopment with significant morbidity and mortality. Poorly drained conditions, empty plastic, glass and other kinds of open containers are common breeding grounds for mosquitoes that transmit malaria in Nigeria. Malaria symptoms include but not limited to moderate to severe shaking chills, high fever, profuse sweating as body temperature falls, general feeling of unease and discomfort (malaise), headache, nausea, vomiting, and diarrhea. Typhoid fever is spread when an individual eats food or drinks water contaminated by human waste. Symptoms of typhoid fever include sudden onset of a fever, severe headache, nausea, etc. Both malaria and typhoid fever are preventable diseases when health and environment are integrated. In Nigeria both poor and rich people are prone to the diseases, due to poor environmental situation. However, the persistent and consistent attack of malaria and typhoid fever on a victim is contingent on the nature of environment and the socio-economic position of the victim. The intention of this study therefore, is to investigate the influence of environment and the socio-economic status of those that are constantly threatened by the diseases in Nigeria, with the overall aim to see how environment and social status affects human health and to proffer solution that will help to maintain a hygienic and clean environment.
\end{abstract}

Key Words: poverty, victim, socio-economic status, environment, malaria fever, typhoid fever

\section{Introduction}

Understanding patterns of health and disease requires that the focus in public health is not only on personal behaviours, biologic traits and specific risks but also on characteristics of the social and physical environments that shape human experience. At the end of the 20th century a challenge of the dominance of biomedicine and the need to give greater emphasis to the ecological context in the study of human health and disease was growing. The emerging new paradigm of public health is based on a holistic conception of health and on creating social and environmental conditions conducive for health. The social and physical surroundings in which people live affect their health. Individuals and populations are embedded within social, political and economic systems that shape behaviours and offer or constrain access to resources necessary to maintain good health. Recognition that health is a product of the antecedent biological evolution interacting with current social and environmental conditions facilitates identification of social and environmental determinants that might be amenable to community interventions and can lead to improved health outcomes.

Attempt to study the issue of poverty has gone beyond theoretical explanation, even though descriptive in nature has practical implication that can be viewed objectively. Poverty is written boldly on the faces of the people it affected. This is because it manifested on physical appearance, their life style, the food they eat, the group they belong to, the type of family structure, their living habit, the nature of environment they live, ill health and access to medical facilities. A universal and simple measure of poverty is to look at different populations' health and life expectancy. It is no surprise that anywhere on earth, poorer communities fare worse than richer ones. Nevertheless, people's health is affected not only by material poverty but also by social exclusion, yet another neglected effect of poverty. Poverty is multidimensional and widely spread in developing societies. Incomes and consumption levels are low and volatile. The available productive assets, particularly for the poor, are few and meagre, increasing vulnerability to shocks. Illiteracy, malnutrition, and ill health are prevalent, with mortality and morbidity rates high.

In Nigeria, low-income communities are the populations living within the worst built environmental conditions. Most solid wastes are disposed on the land in open dumps. Disposal of solid waste on the land without careful planning and management can present a danger to the environment and the human health. An environmental determinant of health is, in general, any external agent (biological, chemical, physical, social, or cultural) that can be causally linked to a change in health status. However, since virtually everything that is not genetically determined could be considered "environmental," this general definition is too all encompassing to be useful. According to World Health Organization (2002), environmental factors play a central role in human health. Human exposures to hazardous agents in the air, water, soil, food also to physical hazards in the environment are major contributors to illness, disability, and death worldwide. Furthermore, deterioration of environmental conditions impedes sustainable development. Poor environmental quality is estimated to be 
directly responsible for approximately 25 percent of all preventable ill health in the world, especially among vulnerable populations.

According to the World Health Organization (WHO, 1992), over one billion people are unable to meet their basic needs (i.e., adequate food, clean water, and shelter) because they lack the necessary income or land. These are environmental hazards that take a far greater toll on human life and suffering in absolute terms. In the developing world, the primary environmental determinants of health are biological agents in the air, water, and soil that account for most deaths and for most deaths in the world. Four million children die annually from diarrheal diseases acquired from contaminated food or water. Over one million people die from malaria each year. Hundreds of millions of people suffer from debilitating intestinal parasitic infestations. Hundreds of millions of people suffer from respiratory diseases caused by biological and chemical agents in the air, both indoors and outdoors.

\subsection{Causes of Malaria fever and Typhoid fever}

Malaria is caused by a parasite called Plasmodium, which is transmitted via the bites of infected mosquitoes. In the human body, the parasites multiply in the liver, and then infect red blood cells. Usually, people get malaria by being bitten by an infective female Anopheles mosquito. Only Anopheles mosquitoes can transmit malaria and they must have been infected through a previous blood meal taken on an infected person. When a mosquito bites an infected person, a small amount of blood is taken in which contains microscopic malaria parasites. About 1 week later, when the mosquito takes its next blood meal, these parasites mix with the mosquito's saliva and are injected into the person being bitten. Because the malaria parasite is found in red blood cells of an infected person, malaria can also be transmitted through blood transfusion, organ transplant, or the shared use of needles or syringes contaminated with blood. Malaria may also be transmitted from a mother to her unborn infant before or during delivery ("congenital" malaria).

Symptoms of malaria include fever and flu-like illness, including shaking chills, headache, muscle aches, and tiredness. Nausea, vomiting, and diarrhea may also occur. Malaria may cause anemia and jaundice (yellow coloring of the skin and eyes) because of the loss of red blood cells. Symptoms usually appear between 10 and 15 days after the mosquito bite. If not treated, malaria can quickly become life-threatening by disrupting the blood supply to vital organs. Infection with one type of malaria, Plasmodium falciparum, if not promptly treated, may cause kidney failure, seizures, mental confusion, coma, and death. In many parts of the world, the parasites have developed resistance to a number of malaria medicines.

Typhoid fever is an acute illness associated with fever that is most often caused by the Salmonella typhi bacteria. It can also be caused by Salmonella paratyphi, a related bacterium that usually leads to a less severe illness. This disease has received various names, such as gastric fever, abdominal typhus, infantile remittent fever, slow fever, nervous fever or pathogenic fever. The name "typhoid" means "resembling typhus" and comes from the neuropsychiatric symptoms common to typhoid and typhus. Despite this similarity of their names, typhoid fever and typhus are distinct diseases and are caused by different species of bacteria. The bacteria are deposited in water or food by a human carrier and are then spread to other people in the area. Typhoid fever is rare in industrial countries but continues to be a significant public-health issue in developing countries, particularly in Nigeria.

The symptoms of typhoid fever usually develop one to two weeks after a person becomes infected with the Salmonella typhi bacteria. If typhoid fever is not treated, the symptoms will develop over four weeks, with new symptoms appearing each week. With treatment, the symptoms should quickly improve within three to five days. Symptoms of typhoid fever during the first week of infection include: a high temperature (fever) that can reach 39-40C (103-104F), which usually increases throughout the day before falling the following morning; abdominal pain; constipation or diarrhea - adults tend to get constipation and children tend to get diarrhea; vomiting - this usually affects children rather than adults; a dry cough; a dull headache that can be felt at the front of the head; severe mental confusion, such as not knowing where you are or what is going on around you; a skin rash made up of pink spots that are $1-4 \mathrm{~cm}$ wide - there are usually fewer than five spots and a feeling of being increasingly very unwell

In the unlikely event that the affected person do not get treatment, the symptoms above will become more severe in the second week. One may experience a swollen abdomen and a slow heartbeat. During the third week, the symptoms of typhoid fever include: loss of appetite; weight loss; physical exhaustion; bouts of foulsmelling, yellow-green, watery diarrhea; severe swelling of the abdomen; rapid breathing; a deterioration of your mental state, such as severe confusion, apathy and, in some cases, psychosis (where a person is unable to tell the difference between reality and their imagination). During the third week, 1 in 10 people will also develop serious complications, such as internal bleeding. 


\subsection{Environmental Determinant of ill Health}

According to WHO, (1987) the environmental factors that majorly affect human health include sources and quality of water supply, sewage disposal methods, housing conditions, food storage methods and hygiene. Indicators for assessing the environmental health status of communities include infant mortality rates, life expectancy rates, access to healthcare, potable water and sanitation and the percentage of the gross national product spent on health. For Nwokoro, (2006) environment is a composite of behavioural settings which greatly affects human health. Environmental factors that affect health are in turn linked to underlying pressures on the environment. Poverty also exerts immense pressure on both the environment and health. The greatest degradation of the urban environment in the Third World is not merely pollution, erosion, mismanagement of waste, and the depletion and deterioration of resources, but rather it is the degraded and immensely impoverished quality of life, particularly for the majority of urban dwellers.

The environment, which sustains human life, is also a profound source of ill health for many of the world's people (WHO, Geneva, 1997). In the least developed countries, one in five children do not live to see their fifth birthday -- mostly because of avoidable environmental threats to health. That translates into roughly 11 million avoidable childhood deaths each year. Hundreds of millions of others, both children and adults, suffer ill health and disability that undermine their quality of life and hopes for the future. These environmental health threats -- arguably the most serious environmental health threats facing the world's population today -- stem mostly from traditional problems long since solved in the wealthier countries, such as a lack of clean water, sanitation, adequate housing, and protection from mosquitoes and other insect and animal disease vectors.

Indeed:

- Contaminated water -- contaminated by faeces, not chemicals -- remains one of the biggest killers worldwide. Lack of adequate water, sanitation, and hygiene is responsible for an estimated 7 percent of all deaths and disease globally, according to one recent estimate. Diarrhea alone claims the lives of some 2.5 million children a year.

- Overcrowding and smoky indoor air -- from burning biomass fuels for cooking or heating -- contribute to acute respiratory infections that kill 4 million people a year, again, mostly children younger than age 5 . The World Bank estimates that between 400 million and 700 million women and children are exposed to severe air pollution, in most instances, from cooking fires.

- Malaria kills 1 million to 3 million people a year, approximately 80 percent of them children. Other mosquito-borne diseases, such as dengue and yellow fever, affect millions more each year and are on the rise, prompting the World Health Organization (WHO) to declare the mosquito "Public Enemy Number One".

In assessing the impact of environment on ill health, Smith (1997) assert that what's more, in many newly and rapidly industrializing regions of the developing world, the populations are in double jeopardy, facing both this unfinished agenda of traditional environmental health problems as well as emerging problems of industrial pollution. Cottage industries, such as backyard tanneries, can place workers and residents in direct contact with hazardous chemicals. In those areas where the use of pesticides and other agricultural chemicals is increasing and safeguards are lax -- or risks poorly understood -- high exposures can ensue, leading to acute poisonings and even death. For countries in the early stages of development, both pesticides and faeces may contaminate drinking-water supplies, and air pollution may stem both from traditional biomass fuels and industrial use of fossil fuels. Such problems are increasingly pronounced in the slum settlements that ring many of the world's cities.

Kristof (1997) is of the view that problems can be particularly acute where economic growth is extremely rapid. In many of the most rapidly developing countries in Asia, industrialization is occurring at triple the pace of the industrial revolution in the West. Many of those regions are also experiencing industrial pollution on a scale not seen in the developed world for the past 40 years -- not since the London fog of 1952 caused some 4,000 excess deaths in the weeks subsequent to the episode. According to the United Nations, cited in Kristof (1997) 13 of the 15 cities with the worst air pollution in the world are in Asia. World Bank (1997), study estimates that more than 2 million people die each year in China alone from the effects of air and water pollution.

In the world's wealthiest regions, such as Europe, North America, and Japan, although environmental risks overall tend to be lower, they have by no means disappeared.

- Asthma is rising dramatically throughout the developed countries, and environmental factors appear to be at least partly to blame.

- Millions of people in Europe and North America are still exposed to unsafe air, and some air pollutants are proving more recalcitrant to control than many expected. 
- Meanwhile, biological contamination is by no means a thing of the past, as shown by the 1993 outbreak of Cryptosporidium in the United States.

- The extension of travel and trade is providing new opportunities for the spread or re-emergence of infectious diseases. In the past two decades, some 30 "new" infectious diseases have emerged (Platt, 1996).

According to Bruce, Lee and Haide (1996) in all regions of the world, populations face the threat of climate change and other global environmental problems, such as stratospheric ozone depletion. Worldwide, fossil fuel emissions continue to rise, bringing with them the risk of climate change and both immediate and long-term health effects. However, it is important to note that although the activities that are driving these changes, such as intense fossil fuel consumption, have largely been concentrated among the wealthiest nations; the impacts are likely to be greatest in the poorest regions that do not have the resources to adapt to them. Similarly, in the wealthiest countries, disadvantaged populations often endure the highest exposures and have the fewest resources to deal with them.

As these examples reveal, despite considerable progress in addressing environmental problems, environmental degradation still poses a huge threat to human health in many regions. The exact nature and scale of environmental risks to health vary dramatically according to where and how one lives. The distribution of risks reflects a number of factors, including the level of socioeconomic development, distribution of wealth, a region's geography and climate (heat and humidity are major killers), and equally important, policy choices and investments.

Furthermore, the outcome of new indicators developed by the World Resources Institute (WRI) indicated that the developing country index examines health risks in three categories: air, water, and food. Some countries, such as poorer countries in Africa, may face high risks from indoor air pollution but low risks from outdoor air pollution; other countries such as India and China face both. In areas where these threats coincide with poor nutrition and/or water-related diseases, the environmental risks to health are likely to be high. Generally, countries in Africa and parts of Asia face the highest environmental threats to health.

In a nutshell, environmental hazards to health fall into two broad categories. The first is a lack of access to essential environmental resources -- chief among them sufficient and clean water, food, shelter, fuel, and air. The second broad category is exposure to hazards in the environment. These hazards include biological agents -- microorganisms such as bacteria and viruses and parasites -- that contribute to the huge global burden of infectious diseases. Biological agents are implicated in diseases from diarrheal to acute respiratory infections, to malaria, to ulcers, and to some cancers. Also included are noxious chemical and physical hazards in the environment. Some pollutants, such as pesticides and industrial solvents, are created by human activities. Others, including arsenic or ultraviolet (UV) radiation, occur naturally in the environment, although exposure can be exacerbated by human activities. These pollutants can undermine health in various ways, by contributing to cancer or birth defects or perhaps by damaging the body's immune system, which renders people more susceptible to a variety of other health risks

\subsection{The Environment of Victim of Malaria and Typhoid Fever in Nigeria}

Healthy populations are prerequisite to sustainable development anywhere in the world, besides, environmental protection is vital to safeguard human health, degraded environment imperil the health of millions, especially of those already vulnerable because of poverty and inequity. Once a person has been exposed to an environmental hazard, health effects manifest themselves which vary in type, intensity and magnitude. The ill-health effects of environmental exposures may be acute or chronic. At this junction the paper explore some environmental conditions that threaten human health particularly within the Nigeria context

The Nigeria urban centres are characterized by two classes of settlements, namely ghettos and Government Residential Areas (GRAs). The GRAs are well planned and the houses are planted and taken care of in the GRAs thus giving rise to beautiful scenery with conducive living conditions. Conversely, the ghettos are characterized by clustered compounds and poorly planned housing system. Stinking gutters, and with surface littered with pieces of solid waste. Traffic congestions are common because of narrow roads and higher population concentrations incidentally this is where the low income earners who are more vulnerable to malaria and typhoid fevers reside.

The drainage systems where the poor and low income earners reside are the open type. Due to poor construction coupled with lack of official specifications on the dimension of the drains, the drains cannot serve their functions effectively. The urban dwellers have the habit of dumping both solid and liquid waste products directly into the drains particularly when it is raining thinking that they will be washed away. When flooding occurs, the dirty contents of drains often empty into nearly houses, roads, wells, streams etc, and causing general pollution.

It is interesting to know that Nigeria does not have a sophisticated technology for solid waste management neither are there any serious measures adapted to check indiscriminate disposal of solid waste in the cities. There are various kinds of solid waste that can be found in the ghettos where the low income earners 
reside. Some of them are bark of agricultural products like sugarcane, groundnuts, peanuts, mangoes, cork of bottles, broken bottles, tiles pieces, polythene, etc. Generally speaking, places where the low income earners reside are dirty and stinking. The outbreaks of diseases such as typhoid fever, malaria, measles, cholera etc, are more frequent and destructive in the ghettos areas than the GRAs. The GRAs are neat and well planned whereas the ghettos where people of lower class concentrate have very poor sanitary conditions. This explains why people residing in this environment are more vulnerable to constant attack of both malaria and typhoid fever. This is because the physical environment that acted as a causal factor has not been taken care of.

The UNEPA (2006) state that wastes that are not properly managed, especially excreta and other liquids and solid wastes from households and the community, are a serious health hazard and could lead to the spreading of diseases. The report further states that unattended wastes lying around attract flies, rats, and other creatures that, in turn, spread diseases. Normally, it is the wet waste that decomposes and releases a bad odour. The bad odour affects the people settled next to the dumpsite, which clearly shows that the dumpsites have serious effects to people settled around or next to them.

In addition to the aforementioned, unsafe drinking water and poor sanitation is another factor that is responsible for constant threat of malaria and typhoid fevers. UNICEF, (2000) posit that contaminated water and inadequate sanitation cause a range of diseases, many of which are life-threatening. The most deadly are diarrheal diseases, 80 percent to 90 percent of which result from environmental factors. In 2001, diarrheal infections caused nearly 2 million deaths in children under age 5, primarily due to dehydration; many more children suffer from nonfatal diarrheal that leaves them underweight, physically stunted, vulnerable to disease, and drained of energy. Poor sanitation conditions and inadequate personal, household, and community hygiene are responsible for most diarrheal infections. Despite significant investments in improving water supplies and sanitation over the last 20 years, about 18 percent of the world's population still lacks access to safe drinking water, and nearly 40 percent have no access to sanitation. More so, United Nations Human Settlement Programme (UNHSP, 2001) declared that at present, people in rural areas are the most affected, although continual urbanization means that increasing numbers of people live in densely populated cities, where they face shortages of potable water supplies and sanitation systems, as well as growing pollution. More than 1 billion people, mostly in Africa, Asia, and Latin America, currently live in slums or as squatters.

UNICEF (2002) maintained that vector-borne diseases, such as malaria, represent an international public health problem, particularly in tropical areas of Africa, Asia, and Latin America. Approximately 1 million children under age 5 in sub-Saharan Africa die of malaria each year; malaria causes about 25 percent of all deaths among children in the region, especially among children living in remote rural areas with poor access to health services. Malaria also contributes to low birth weight, one of the leading risk factors for infant mortality, because pregnant women are more susceptible to both malaria and anaemia.

\subsection{Poverty and ill Health}

According to Thomas J. (2009) poverty is defined as the condition of having insufficient resources or income. In its most extreme form, poverty is a lack of basic human needs, such as adequate and nutritious food, clothing, housing, clean water, and health services. Extreme poverty can cause terrible suffering and death, and even modest levels of poverty can prevent people from realizing many of their desires. The world's poorest people - many of whom live in developing areas of Africa, Asia, Latin America, and Eastern Europe - struggle daily for food, shelter, and other necessities. They often suffer from severe malnutrition, epidemic disease outbreaks, famine, and war. The reasons for poverty are not clear. Some people believe that poverty results from a lack of adequate resources on global level — resources such as land, food, and building materials - that are necessary for the well-being or survival of the world's people. Others see poverty as an effect of the uneven distribution of resources around the world on an international or even regional scale. This second line of reasoning helps explain why many people have much more than they need to live in comfort, while many others do not have enough resources to live.

Health is a state of complete well-being: physical, mental, and emotional. This definition emphasizes the importance of being more than disease free, and recognizes that a healthy body depends upon a healthy environment and a stable mind. The concept of illness includes those conditions often accompanied by pain or discomfort that inhibit a human being's ability to physically, mentally, or emotionally perform in a normal and proper manner. In most cultures when people need assistance in maintaining their health, dealing with illness, or coping with problems related to health and illness, they seek assistance from personnel specialized in the fields of health care.

In most cases the poor suffer worse health and die younger. They have higher than average child and maternal mortality, higher levels of disease, more limited access to health care and social protection, and gender inequality disadvantages further the health of poor women and girls. The reason for these is that the poor people live in unsafe and overcrowded housing, which lacks access to safe water, or to excreta disposal. They are much more likely than the wealthy to be exposed to pollution (both indoors and outdoors), traffic, industry (associated with both the formal and informal sector), and other risks at home, work, and in their communities. They are 
more likely to consume insufficient food, or food of poor quality, making them even more vulnerable to the illhealth effects from environmental exposures. The urban poor frequently suffer from a double burden of disease, with problems related to poverty and a lack of basic services, as well as with the impact on health of large scale and rapid industrialisation, urbanisation and technological development. Indeed, it is often difficult to distinguish traditional risks from new and emerging risks associated with industrialisation. For example pesticides and faeces may contaminate the same water supplies. Air pollution may stem simultaneously from burning dirty household fuels and industrial use of fossil fuels. Nowhere is this duality of health and environmental burdens more evident than in low-income areas on the peripheries of cities.

It is well documented that exposures related to inadequate water and sanitation, excreta disposal, polluted air, particularly indoors from cooking and heating fuels used, are associated with a wide range of diseases, both infectious (ARI, diarrhoea, vector-borne diseases) and non infectious. Diarrhoeal diseases are 5 to 6 times more common in developing countries than in developed countries (WHO 1997). Such diseases are closely related to poor sanitation and hygiene, and the resultant contamination of food and water. It is estimated that upwards of 1100 million people lack adequate and safe drinking water, and around three times this many people lack adequate sanitation (WHO 1997).

More so, Phipps (2003) revealed that research focusing on individuals has found a very robust relationship between an adult individual's income and that individual's health, using a range of measures for both. Regardless of how measures of health status and measures of SES are combined, there is little doubt that poverty leads to ill health. For example, in a recent review of the literature, Benzeval and Judge cited in Phipps (2003) provide evidence from 16 studies using eight different data sets from four different countries. Health status outcome measures include: subjective self-reports, mortality, emotional stability, chronic conditions, general life satisfaction and physical functioning. Socio-economic status measures include: current income level, recent income change, poverty flags, current earnings, multi-period averaged incomes, relative position in the income distribution and number of spells of poverty. In summing up their review, the authors conclude: All of the studies that include measures of income level find that it is significantly related to health outcomes.

According to Phipps (2003) in a similarly work conducted by Mullahy and colleagues (2001) conclude: Voluminous empirical studies and reviews demonstrate a robust association between income and morbidity and mortality, using various measures of both income and health, across samples, and at various time points. An important research issue in the study of poverty and health is the possibility for ill health to limit an individual's ability to engage in paid work and hence reduce his or her income, even if he or she comes from an affluent background. This possibility is variously referred to as reverse causation, health selection or endogeneity. The problem is that if all we know is that ill health and low income are often observed together, we cannot sort out which caused which. However, the studies reviewed by Benzeval and Judge all used longitudinal data so that they could follow individuals over time rather than merely observing relationships at a point in time. Most of the studies reviewed tried to control for the possibility that ill health causes low income rather than that low income causes ill health61; all conclude that reverse causation is not a serious problem and that the main direction of influence is from poverty to poor(er) health.

Further important conclusions from this body of work include the following:

- The relationship between individual income and health is non-linear (i.e. low-income individuals suffer larger negative health consequences than high-income individuals reap health benefits, though highincome individuals $d o$ reap benefits).

- Longer-term measures of average income have larger associations with health than measures of current income, which can be highly volatile.

Long-duration poverty has larger (negative) health consequences than occasional episodes of poverty.

- Both income level and income changes are significant predictors of health status, but income level is the more important of the two.

- Negative shocks to income are more important for health than positive shocks

\subsubsection{Theoretical Frame-work}

According to Kerlinger (1986) cited in Donald et-al (1996) defines theory "as a set of interrelated constructs (concepts), definition, and prepositions that present a systematic view of phenomena by specifying relations among variables with the purpose of explaining and predicting the phenomena". Theories knit together the result of observations, enabling the scientists to make general statements about variables and the relationships among variables. The theory adopted in this work to show the relationship between human environment and ill health is situated within the frame work of ecological theory.

\subsubsection{Ecological Theory of Health}

This work is guided by ecological theory. The heart of ecological theory is the natural ecosystem. By analogy, concepts from natural systems are used to help understand human systems and environments. The 
ecological approach to the study of human health made on analogy between environment and human organism, the ecology is a branch of biology that is concern with the behaviour of living things in their relation to the environment. For them, society is first like a human organism, the relationship that constitutes a society is so intimate and necessary so that any influence felt at one point is almost, immediately transmitted throughout, to other parts of the society. Ecological theorist asserts that human health is not an isolated phenomenon. In the first place, it is fostered by certain kinds, of social environments, including in that term a whole variety of political, social, economic, ethical factors, etc. One of the major concerns of ecological theory is the study of aspects of the physical environment and their implications for the distribution of human disease. Traditionally, the concerns of environmental epidemiology included the contamination of air, water, and food. However the focus now includes climate change, and other large scale environmental change related to globalization and urbanization. Such environmental changes, particularly as they coincide with particular demographic shifts (for example, shifts in birth rate) have implications for a number of adverse health outcomes including cancers, reproductive problems, and infectious diseases.

\subsubsection{Conclusion and Recommendation}

This paper maintained that social and environmental conditions shape patterns of population health across the world. While diseases are influenced by a number of specific risk factors and agents, socioeconomically disadvantaged men and women and those who are socially excluded from full participation in their societies are almost without exception at increased risk for whatever diseases are prevalent in their society. Thus, while specific disease agents and risk factors come and go, the social and physical environment shapes both the distribution of risks as well as susceptibility and resilience to risk. Poverty, economic inequality, social isolation and exclusion and job stress along with environmental conditions related to pollution, unsanitary living conditions and poor nutrition shape health in various ways that the health care system will be hard pressed to fix. Poor water supply, sanitation and hygiene are another environmental source of ill-health. Inadequate sewage treatment and poor sanitation result in diarrhoeal diseases caused by bacteria, such as cholera or E. coli, by viruses such as norovirus or rotavirus or protozoan parasites such as cryptosporidiosis or giardiasis. The greatest health risk in this area comes from unsafe drinking water. This study concludes that environmental problems can have a substantial impact on human health. Unsafe water supply, sanitation and hygiene are responsible for typhoid and malaria fever in Nigeria and in most cases affected the health of the poor people.

\subsubsection{Recommendation}

This study has clearly shown there is a link between material inequalities and ill health in which the poorer groups suffer higher levels of ill health. This is because poverty determine where people resides especially in an environment that lack certain basic necessities that are useful for people's wellbeing. Looking at these issues this study therefore recommends the following as regards to Nigeria situations:

- There is need to drastically reduce the level of poverty.

- Hygienic awareness must be created in the community.

- Underground drainage system, toilets must be constructed.

- Clean and portable water should be provided.

- Functional refuse collection and dumping site should be created.

\section{References}

[1] Anne E. Platt (1996) “Infecting Ourselves: How Environment and Social Disruptions Trigger Disease,” World Watch paper 129 (World Watch Institute, Washington, D.C.

[2] Donald, Ary et-al (1996). Introduction to Research in Education.5th Edition. Harcourt Brace College Publishers

[3] H. Gelband and Strans Field (2001) "The Evidence Base for Interventions to Reduce Under-Five Mortality in Low - and Middle Income Countries”. CMH Working paper Series, number WG5:9 (Geneva: WHO Commission on Macro Economics and Health).

[4] James P. Bruce, Hoesung Lee, and Erik F. Haites, (1996), Economic and Social Dimensions of Climate Change. Cambridge University Press.

[5] Kirk R. Smith (1997) “Development, Health and the Environmental Risk Transition” In G.S Shahi et al., eds, International Perspective on Environment, Development and Health. Springer publishing company, New York.

[6] Nicolas D. Kristof (1997) “Across Asia, A Pollution Disaster Hovers.” The New York Times (Nov, 28, 1997)

[7] UNEPA (2006) Informal Solid Waste Management. Http://www.unep.org/pdf/kenyawastemngtsector/sector/chapter1.pdf

[8] UNICEF et al.,(2000). "Global Water Supply and Sanitation Assessment”. Data accessed online@, www.childinfo.org/eddb/water.html on Sept. 2011

[9] UNICEF (2002) "Malaria Statistics". Data accessed online @ www.childinfo.org/eddb/malaria/index.html, on Sept.2012

[10] United Nations Human Settlement Programme (2001). "The State of The World's Cities, Nairobi, Kenya".

[11] WHO, Geneva (1996) The World Health Report: Fighting Diseases, Fostering Development.

[12] WHO, Geneva (1997) Health and Environment in Sustainable Development: Five Years after the Earth Summit. 